\title{
Efficient isolation inner cell mass from blastocysts by improved microsurgical technique
}

\author{
Yan Ding ${ }^{1}$, Yi Gan ${ }^{1}$, Jing Bo Feng ${ }^{1}$, Shao Hua $\mathrm{Qi}^{1}$, Rui Ming $\mathrm{Li}^{1}$, Dong Sheng $\mathrm{Li}^{1}$ \\ ${ }^{1}$ Hubei Provincial Key Laboratory of Embryonic Stem Cell Research, Taihe Hospital, Yunyang Medical College,32 S. Renmin \\ Road, Shiyan 442000, China
}

Embryonic stem (ES) cells, derived from the inner cell mass (ICM) of a blastocyst, can differentiate into almost all kinds of cells of the body under certain circumstances. These so-called pluripotent cells have great potential in cell therapy treating many intractable human diseases. However, its derivation is by no means an easy task. Isolation of ICM is one of the crucial steps of the procedure. Most currently-existed ES cells were derived from the ICM isolated from a blastocyst by immunosurgery. This technique is not only time-consuming, but also has a danger contaminating ES cells with animal materials, which may cause unpredicted diseases if transplanted into human being in cell therapy. The authors invented a new method of isolation by microsurgical technique with the aid of an inverted microscope (Olympus IX71) equipped with micromanipulators (Eppendorf Transferman NK2). This method has three steps: 1) Holding needle anchored the embryo on the side of ICM with suction supplied by a micrometer syringe; 2) cutting off the zona pellucida, and 3) tearing off the trophoblastic layer from the nudity blastocyst. They compared their method with traditional immunosurgery in isolating time, ICM adherence time, as well as the successful rates of derivation. ICR mouse blastocysts of 3.5dpc were harvested by means of superovulation. Fifty of Grade blastocysts were randomly divided into the microsurgery group and the immunosurgery group, 25 each. The isolated ICM from both methods were cultured on the mouse embryonic fibroblast (MEF) cell feeder layers for 3 to 5 days. The ES cell-like colonies were sub-cloning cultured twice and further passaged. The pluripotent properties of the cell lines obtained were analyzed on the basis of their alkaline phosphatase activity and their capacity to form complex embryonic bodies with rhythmically contracting cardiomyocytes. In results, using this improved micromanipulation procedure, the adherence time is almost the same with immunosurgery method, but the adherence and success rate of establish embryonic stem cell lines were greatly increased compared with immunosurgery method. Thirteen stable ES clones were obtained and were passaged to 13th generation. These cell lines were characterized as nest like in morphology, positive for alkaline phosphatatse staining and expression of markers of undifferentiated mES cells, keep normal karyotype. The differentiation of ES cells were outgrowth from embryonic bodies in vitro, while three embryonic derms were formed from ES cells in vivo.

In conclusion, this improved microsurgical method of ICM isolation is more efficient and super in derivation of ES cells. Keywords: ES cell, ICM, Micromanipulation, microimmunosurgery, blastocyst

Cell Research (2008) 18:s39. doi: 10.1038/cr.2008.129; published online 4 August 2008

Correspondence: Dong Sheng Li

E-mail: dsli@yymc.edu.cn 